\title{
Effect of Different Coating Elements on Shelf Life of Indian Olive (Elaeocarpus floribundus Blume)
}

\author{
Sukanya Misra ${ }^{1 *}$, Priyam Chattopadhayay ${ }^{2}$ and Prodyut kr. Paul ${ }^{1}$
}

${ }^{1}$ Dept. of Pomology and Post Harvest Technology, UBKV, Pundibari, Cooch Behar, W.B. (736 165), India

${ }^{2}$ Dept. of Horticulture and Post Harvest Technology, Palli Siksha Bhavana, Visva- Bharati, Sriniketan, West Bengal (731 236), India

\section{Corresponding Author}

Sukanya Misra

e-mail: sukanyamisra28@gmail.com

\author{
Article History \\ Article ID: AR1893 \\ Received in $15^{\text {th }}$ August, 2018 \\ Received in revised form $23^{\text {rd }}$ February, 2019 \\ Accepted in final form $28^{\text {th }}$ February, 2019
}

\begin{abstract}
The experiment was conducted during 2015-16 in the laboratory of Department of Pomology and Postharvest Technology, Horticulture, Uttar Banga Krishi Viswavidyalaya to study the effect of different Coating elements on shelf life of Indian Olive (Elaeocarpus floribundus Blume). Among 13 different treatments, spoilage \% was minimum in Guar gum and highest (65\%) occurred in Control. At the end of storage weight loss of chitosan treated fruits were lower than the other treatments, in control it was $47.09 \%$. A combination treatment of paraffin liquid and calcium chloride and uncoated fruits resulted in highest disease incidence (30\%). At the last day of storage lowest (6.04\%) total sugar percentage was seen in Control whereas highest (6.29\%) was seen in $\mathrm{CaCl}_{2}$ and Guar gum treated fruit. Reducing sugar was found lowest $(3.19 \%)$ in Calcium chloride (2\%) $(\mathrm{w} / \mathrm{v})+$ Paraffin liquid $(5 \%)(\mathrm{v} / \mathrm{v})$ and Calcium chloride $(2 \%)(\mathrm{w} / \mathrm{v})+$ Chitosan $(2 \%)(\mathrm{w} / \mathrm{v})$, where as highest reducing sugar was seen in Calcium chloride $(1 \%)(\mathrm{w} / \mathrm{v})+$ Paraffin liquid $(5 \%)(\mathrm{v} / \mathrm{v})$ at the final day of storage. The maximum $(3.01 \%)$ non-reducing sugar was found in Gum tragacanth (2\%) followed by Calcium chloride (2\%) (w/v)+Paraffin liquid (5\%)(3.01\%) and the lowest (2.74\%) was seen in Calcium chloride (1\%)(w/v)+Paraffin liquid (5\%)(v/v). Ascorbic acid was highest (20.86 g $100 \mathrm{~g}^{-1}$ of pulp) in Calcium chloride (2\%) (w/v)+Chitosan (2\%)(w/v) and Calcium chloride (2\%) (w/v)+Parafin liquid and it was lowest (18.2 $\mathrm{g} 100 \mathrm{~g}$ of pulp $\left.{ }^{-1}\right)$ in Control. The lowest (1.25\%) acid content was present in Calcium chloride (2\%) (w/v)+Guar gum (2\%) (w/v), highest $(1.93 \%)$ was seen in Calcium chloride (1\%) (w/v)+Chitosan (2\%)(w/v). Maximum (387.633 g) penetration force was seen in Calcium chloride (1\%) (w/v)+Guar gum (2\%) $(\mathrm{w} / \mathrm{v})$ and minimum $(241.067 \mathrm{~g})$ was in Control.
\end{abstract}

Keywords: Indian Olive, shelf life, coating element, Chitosan

\section{Introduction}

Indian olive or jalpai (Elaeocarpus floribundus Blume), of Elaeocarpaceae family is a minor fruit crop. In spite of having nutritional value, the crop has not been properly known to the people. Jalpai is a medium to tall tree. Flowers appear during April-May and fruits mature for harvest in October-November (Sankarsan et al., 2006). Fruits are greenish in colour, single seeded, the shape resembles olive fruit, outer surface contain fleshy mesocarp. There is a good demand for matured fruit in the local market for the preparation of pickles and chutney (Bhowmick, 2011). The total area under fruit crops is 6428 ha and production is $9488 \mathrm{t}$ in India (Anonymous, 2018), out of which the northern parts of West Bengal comprises around $33 \%$ area and $36 \%$ of production. Jalpai (Indian olive) is one of the important fruit crop of this region, mainly grown as homestead crops in shady or semi-shady condition with no or very few management practices. Jalpai fruits have high nutritional value, it contains $0.69 \%$ protein, carbohydrates $19.5 \%$, mineral matter $0.59 \%$, vitamin C content of dry leaves is $257 \mathrm{mg} 100$ $\mathrm{g}^{-1}$. The fruits are used in dysentery and diarrhoea. The leaves are used in rheumatism and as an antidote for poison (De and Parikh, 1985).

Perishability is one the main issues in post-harvest handling and marketing of fresh fruits and vegetables. In case of jalpai fruit, its short shelf-life (6 to 7 days) limits somewhat its marketability. Numerous technologies have been developed as means to extend their shelf-life and eating quality, among them use of edible coatings is a technology which is applied on many fruits to control moisture transfer, gas exchange or oxidation processes. Edible coatings can also provide an additional protective coating to fruits and can also give the same effect as modified atmosphere storage in modifying internal gas composition (Dhall et al., 2012). Edible coatings are biodegradable, environmentally friendly, economic, consumer compatible method of storage. The ingredients used in edible coatings are obtained from natural sources which provide several advantages over synthetic material (Tharanathan, 2003). Coating is basically a thin layer of edible polymeric materials directly applied on the surface of food items such as fresh fruits and vegetables. Due presence of 
high moisture content, Marketability of most of the fruits is lost very rapidly after harvesting due to quick desiccation, discolouration, disease incidence and spoilage of fruits. In order to have good return and to avoid market glut it become essential to store the fruits for a considerable period. Colossal waste of fruits in storage occurs due to improper harvesting, occurrences of diseases during the storage and transit and also due to improper technical knowledge. Fruits can fetch better price if ripening can be adjusted with market demands and stored properly.

The principal aim of storage is to check the rate of transpiration, respiration and diseases infection. These can be achieved by the use of different coating materials and treatment with other chemicals. Keeping the above point in view, on the storage life of Indian Olive and physico-chemical changes during storage.

\section{Materials and Methods}

The present lab investigation was done in the laboratory of Department of Pomology and Postharvest Technology, Faculty of Horticulture, Uttar Banga Krishi Viswsavidyalaya, Pundibari, Coochbehar, West Bengal, India during the year 2015-16. Fully mature Jalpai fruits were collected from orchard of the University. The treatments are $\mathrm{T}_{1}$ : Guar gum (2\%) (w/v); $\mathrm{T}_{2}$ : Calcium chloride (1\%) (w/v) +Guar gum (2\%) (w/v); $\mathrm{T}_{3}$ : Calcium chloride (2\%) (w/v)+Guar gum (2\%) (w/v); $\mathrm{T}_{4}$ : Paraffin liquid $(5 \%)(v / v) ; T_{5}$ : Calcium chloride (1\%)(w/v)+Paraffin liquid (5\%) $(\mathrm{v} / \mathrm{v}) ; \mathrm{T}_{6}$ : Calcium chloride (2\%)(w/v)+Paraffin liquid (5\%)(v/v); $\mathrm{T}_{7}$ : Gum tragacanth (2\%) (w/v); $\mathrm{T}_{8}$ : Calcium chloride (1\%) $(\mathrm{w} / \mathrm{v})+$ Gum tragacanth $(2 \%)(\mathrm{w} / \mathrm{v}) ; \mathrm{T}_{9}$ : Calcium chloride $(2 \%)$ (w/v)+Gum tragacanth(2\%) (w/v); $\mathrm{T}_{10}$ : Chitosan (2\%) (w/v); $\mathrm{T}_{11}$ : Calcium chloride (1\%) (w/v)+Chitosan (2\%) (w/v); $\mathrm{T}_{12}$ : Calcium chloride (2\%) (w/v)+Chitosan (2\%) (w/v); $T_{13}$ : Control. Observations were recorded at an interval of 3 days during storage period. The observations are physical parameters i.e. fruit retention percentage, physiological loss in weight, disease incident percentage, fruit color. After harvesting of the fruits, TSS (Mazumdar and Majumder, 2003), total sugar and reducing sugar (Mazumdar and Majumder, 2003), nonreducing sugar (Mazumdar and Majumder, 2003), titratable acidity (Rangana, 1977), ascorbic acid (Rangana, 1977) and Penetration force were estimated.

Analysis of variance (one way classified data) for each parameter was performed using ProcGIm of Statistical Analysis System (SAS) software (version 9.3). Mean separation for different treatment under different parameter were performed using Least Significant Difference (LSD) test ( $p \leq$ 0.05). Data transformation was done as per the standard rules (Gomez and Gomez, 1983).

\section{Results and Discussion}

\subsection{Effect of different treatments on spoilage percentage} during storage of Indian Olive

The result of which is presented below-Spoilage of fruits occurred due to excess loss of moisture and disease attack. At first day of storage there was no spoilage of fruits. After 3 days of storage, spoilage of fruits was lowest in guar gum treatment $\left(T_{1}\right)$ than the other treatments. Spoilage per cent was also low in paraffin liquid and calcium chloride combined treatment $\left(T_{5}\right)$. Spoilage per cent was highest $(13.33 \%)$ in the treatments with gum tragacanth $\left(T_{7}\right)$ and calcium chloride-chitosan combined treatment $\left(T_{11}\right)$. On $6^{\text {th }}$ day, after storage, spoilage was found to progress further in all treatments. Highest spoilage occurred in chitosan treated fruits $\left(T_{10}\right)(26.66 \%)$. Spoilage \% was lowest (8.33\%) in guar gum treated $\left(T_{1}\right)$ fruits than the other treatments. After 9 days of storage, it was seen that spoilage $\%$ was highest in fruits under control $\left(T_{13}\right)$. It was above $35 \%$ and was about three times more than guar gum treatment $\left(T_{1}\right)(11.66 \%)$. After 12 days of storage again spoilage $\%$ was highest in $\mathrm{T}_{13}$ with $51.66 \%$ fruits were found to be rotten. Lowest spoilage per cent occurred in guar gum treated fruits. $\left(T_{1}\right)(20 \%)$. At the end of storage, spoilage per cent was minimum in guar gum treated fruits $\left(T_{1}\right)$ and highest $(65 \%)$ occurred in uncoated fruits $\left(T_{13}\right)$. Table 1 represented that Spoilage was maximum in fruits under control due to microbial attack, mainly fungal growth, high respiration, and oxidation as compared to coated fruits.

Table 1: Effect of different treatments on spoilage \% during storage of Indian Olive

\begin{tabular}{|c|c|c|c|c|c|}
\hline \multirow{2}{*}{$\begin{array}{l}\text { Treat- } \\
\text { ments }\end{array}$} & \multicolumn{5}{|c|}{ Days after storage } \\
\hline & 3 & 6 & 9 & 12 & 15 \\
\hline $\mathrm{T}_{1}$ & $1.66^{c}$ & $8.33^{c}$ & $11.66^{f}$ & $20.00^{f}$ & $23.33^{d}$ \\
\hline $\mathrm{T}_{2}$ & $6.66^{\mathrm{abc}}$ & $11.66^{c}$ & $16.66^{\mathrm{ef}}$ & $23.33^{e f}$ & $33.33^{c}$ \\
\hline $\mathrm{T}_{3}$ & $8.33^{a b c}$ & $15.00^{\mathrm{bc}}$ & $21.66^{\text {de }}$ & $26.66^{\text {def }}$ & $31.66^{\mathrm{cd}}$ \\
\hline $\mathrm{T}_{4}$ & $6.66^{\mathrm{abc}}$ & $13.33^{b c}$ & $20.00 d^{e f}$ & $26.66^{\text {def }}$ & $33.33^{c}$ \\
\hline $\mathrm{T}_{5}$ & $3.33^{b c}$ & $16.66^{\mathrm{abc}}$ & $20.00 d^{\text {ef }}$ & $30.00^{\text {de }}$ & $31.66^{\mathrm{cd}}$ \\
\hline $\mathrm{T}_{6}$ & $10.00^{\mathrm{ab}}$ & $11.66^{c}$ & $18.33^{\text {ef }}$ & $23.33^{\mathrm{ef}}$ & $33.33^{c}$ \\
\hline $\mathrm{T}_{7}$ & $13.33^{a}$ & $23.33^{\mathrm{ab}}$ & $36.66^{\mathrm{ab}}$ & $46.66^{\mathrm{ab}}$ & $43.33^{b}$ \\
\hline $\mathrm{T}_{8}$ & $10.00^{\mathrm{ab}}$ & $16.66^{\mathrm{abc}}$ & $28.33^{b c d}$ & $33.33^{c d}$ & $45.00^{b}$ \\
\hline $\mathrm{T}_{9}$ & $8.33^{a b c}$ & $23.33^{\mathrm{ab}}$ & $35.00^{\mathrm{ab}}$ & $41.66^{b c}$ & $46.66^{b}$ \\
\hline $\mathrm{T}_{10}$ & $8.33^{\mathrm{abc}}$ & $26.66^{a}$ & $33.33^{\text {ef }}$ & $43.33^{\mathrm{ab}}$ & $46.66^{b}$ \\
\hline $\mathrm{T}_{11}$ & $13.33^{\mathrm{a}}$ & $23.33^{\mathrm{ab}}$ & $40.00^{a}$ & $50.00^{\mathrm{ab}}$ & $51.66^{b}$ \\
\hline $\mathrm{T}_{12}$ & $5.00^{\mathrm{bc}}$ & $16.66^{a b c}$ & $25.00^{\text {cde }}$ & $33.33^{c d}$ & $43.33^{b}$ \\
\hline $\mathrm{T}_{13}$ & $10.00^{\mathrm{ab}}$ & $23.33^{\mathrm{ab}}$ & $38.33^{a}$ & $51.66^{a}$ & $65.00^{a}$ \\
\hline SEm \pm & 2.401 & 3.100 & 2.849 & 2.811 & 2.734 \\
\hline $\begin{array}{l}C D \\
(p=0.05)\end{array}$ & 6.982 & 9.014 & 8.283 & 8.173 & 7.949 \\
\hline
\end{tabular}

3.2. Effect of different treatments on physiological loss in weight during storage of Indian Olive.

The data regarding the physiological loss in weight during storage period was observed at 3,6,9,12 and 15 days interval. At the end of storage it was concluded from Table 
2 that weight loss of chitosan treated $\left(T_{10}, T_{11}, T_{12}\right)$ fruits was lower than the other treatments. Under control $\left(T_{13}\right)$ it was $47.09 \%$. During storage of Indian olive various observations were taken, physiological loss in weight was one of them. During storage, physiological loss in weight is a common phenomenon, Moisture and subsequent weight loss in fruits increased linearly with increase in storage duration due to water loss and respiration (Ghafiret al., 2009). In coated fruits reduction in weight was lower than uncoated fruits, it is due to effects of these coatings as a semi permeable barrier against oxygen, carbon dioxide, moisture and solute movement, thus reducing respiration, water loss and oxidation reaction rates (Baldwin et al., 1999).

\begin{tabular}{|c|c|c|c|c|c|}
\hline \multirow{2}{*}{$\begin{array}{l}\text { Treat- } \\
\text { ments }\end{array}$} & \multicolumn{5}{|c|}{ Days after storage } \\
\hline & 3 & 6 & 9 & 12 & 15 \\
\hline $\mathrm{T}_{1}$ & $6.90^{\text {bcde }}$ & $13.94^{b c}$ & $23.00^{\mathrm{bcd}}$ & $29.13^{b c}$ & $35.17^{b c d}$ \\
\hline $\mathrm{T}_{2}$ & $5.61^{\text {cde }}$ & $9.95^{d}$ & $20.36^{\mathrm{bcd}}$ & $27.21^{\mathrm{bcd}}$ & $34.64^{\text {bcde }}$ \\
\hline $\mathrm{T}_{3}$ & $7.28^{\text {bcde }}$ & $12.58^{\mathrm{bc}}$ & $23.68^{b c}$ & $29.10^{b c}$ & $37.12^{b c}$ \\
\hline $\mathrm{T}_{4}$ & $5.97^{\text {cde }}$ & $13.72^{b c}$ & $21.26^{\mathrm{bcd}}$ & $30.07^{b}$ & $37.16^{b c}$ \\
\hline $\mathrm{T}_{5}$ & $8.68^{b c d}$ & $14.37^{b c}$ & $22.39^{\mathrm{bcd}}$ & $30.04^{b}$ & $36.11^{b c}$ \\
\hline $\mathrm{T}_{6}$ & $7.68^{\text {bcde }}$ & $13.79^{b c}$ & $22.40^{\mathrm{bcd}}$ & $30.35^{b}$ & $36.84^{b c}$ \\
\hline $\mathrm{T}_{7}$ & $9.16^{b c}$ & $17.24^{b}$ & $23.41^{b c}$ & $32.09^{b}$ & $39.56^{\mathrm{ab}}$ \\
\hline $\mathrm{T}_{8}$ & $8.15^{\text {bcde }}$ & $15.63^{b c}$ & $23.58^{b c}$ & $31.13^{b}$ & $38.01^{b}$ \\
\hline $\mathrm{T}_{9}$ & $10.91^{b}$ & $16.99^{b}$ & $24.86^{b}$ & $33.30^{\mathrm{b}}$ & $41.10^{\mathrm{ab}}$ \\
\hline $\mathrm{T}_{10}$ & $4.22^{\mathrm{e}}$ & $9.52^{d}$ & $15.21^{\mathrm{e}}$ & $20.86^{e}$ & $26.13^{e}$ \\
\hline $\mathrm{T}_{11}$ & $4.96^{\text {cde }}$ & $10.50^{\mathrm{bc}}$ & $15.88^{\mathrm{cd}}$ & $21.13^{\mathrm{cd}}$ & $28.62^{\text {cde }}$ \\
\hline $\mathrm{T}_{12}$ & $4.59^{\text {de }}$ & $10.49^{b c}$ & $16.18^{\text {cd }}$ & $20.57^{e}$ & $26.69^{\text {de }}$ \\
\hline $\mathrm{T}_{13}$ & $15.37^{\mathrm{a}}$ & $23.69^{a}$ & $35.19^{a}$ & $41.25^{\mathrm{a}}$ & $47.09^{a}$ \\
\hline SEm \pm & 1.30 & 2.00 & 2.34 & 2.55 & 2.74 \\
\hline $\begin{array}{l}C D \\
(p=0.05)\end{array}$ & 3.80 & 5.83 & 6.82 & 7.41 & 7.97 \\
\hline
\end{tabular}

3.3. Effect of different treatments on disease incidence during storage of Indian olive

Disease incidence mainly occurred due to fungal and mould growth. It is another cause of post harvest loss. It was minimum after 3 days of storage, and gradually increased as the storage period advanced. At the end of 15 days storage, it was observed from Figure 1. that combination treatment of paraffin liquid and calcium chloride $\left(\mathrm{T}_{5}\right)$ and uncoated fruits $\left(T_{13}\right)$ resulted in highest disease incidence $(30 \%)$.

3.4. Effect of different treatments on colour change during storage of Indian olive

Observation during storage of Indian olive revealed that fruits colour of Indian olive was changed from yellowish green to greenish brown (Table 3 ). At the time of harvesting

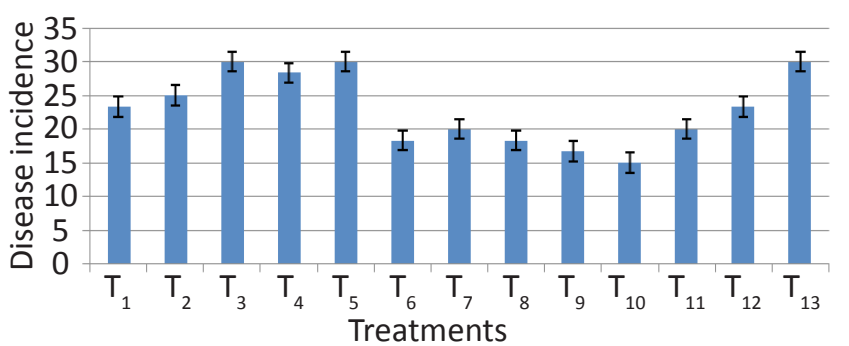

Figure 1: Effect of different treatments on disease incidence during storage of Indian olive

\begin{tabular}{|c|c|c|c|c|c|c|}
\hline \multirow{2}{*}{$\begin{array}{l}\text { Treat- } \\
\text { ments }\end{array}$} & \multicolumn{5}{|c|}{ Days after treatments } & \multirow[b]{2}{*}{15} \\
\hline & 0 & 3 & 6 & 9 & 12 & \\
\hline \multirow[t]{2}{*}{$\mathrm{T}_{1}$} & Y-G-G & Y-G-G & Y-G-G & Y-G-G & Y-G-G & $Y-G-G$ \\
\hline & $144 \mathrm{C}$ & $144 \mathrm{~A}$ & 146 B & $146 \mathrm{~A}$ & 148 B & 147 B \\
\hline \multirow{2}{*}{$\mathrm{T}_{2}$} & Y-G-G & Y-G-G & Y-G-G & Y-G-G & Y-G-G & $Y-G-G$ \\
\hline & $144 \mathrm{C}$ & $144 \mathrm{~B}$ & $146 \mathrm{~A}$ & $146 \mathrm{~A}$ & $148 \mathrm{~A}$ & $148 \mathrm{~A}$ \\
\hline \multirow{2}{*}{$\mathrm{T}_{3}$} & Y-G-G & Y-G-G & Y-G-G & Y-G-G & Y-G-G & $Y-G-G$ \\
\hline & $144 \mathrm{C}$ & $144 \mathrm{~A}$ & $146 \mathrm{C}$ & $146 \mathrm{~A}$ & 148 B & 147 B \\
\hline \multirow{2}{*}{$\mathrm{T}_{4}$} & Y-G-G & Y-G-G & Y-G-G & Y-G-G & Y-G-G & $Y-G-G$ \\
\hline & $144 \mathrm{C}$ & 144B & $146 \mathrm{C}$ & $146 \mathrm{~B}$ & $148 \mathrm{~A}$ & $147 \mathrm{~A}$ \\
\hline \multirow[t]{2}{*}{$\mathrm{T}_{5}$} & Y-G-G & Y-G-G & Y-G-G & Y-G-G & Y-G-G & $Y-G-G$ \\
\hline & $144 \mathrm{C}$ & $144 \mathrm{~A}$ & 146 B & $146 \mathrm{~A}$ & 148 B & $147 \mathrm{~B}$ \\
\hline \multirow[t]{2}{*}{$\mathrm{T}_{6}$} & Y-G-G & Y-G-G & Y-G-G & Y-G-G & Y-G-G & $Y-G-G$ \\
\hline & $144 \mathrm{C}$ & $144 \mathrm{~A}$ & $146 \mathrm{C}$ & $146 \mathrm{~B}$ & $148 \mathrm{~A}$ & $148 \mathrm{~A}$ \\
\hline \multirow[t]{2}{*}{$\mathrm{T}_{7}$} & Y-G-G & Y-G-G & Y-G-G & Y-G-G & Y-G-G & $Y-G-G$ \\
\hline & 144 C & $144 \mathrm{~A}$ & 146 B & 146 B & 148 B & $148 \mathrm{~A}$ \\
\hline \multirow[t]{2}{*}{$\mathrm{T}_{8}$} & Y-G-G & Y-G-G & Y-G-G & Y-G-G & Y-G-G & $G-G-$ \\
\hline & $144 \mathrm{C}$ & 144 B & 146 B & 146 B & $148 \mathrm{~A}$ & N137 C \\
\hline \multirow[t]{2}{*}{$\mathrm{T}_{9}$} & Y-G-G & Y-G-G & Y-G-G & Y-G-G & G-G-G & $Y-G-G$ \\
\hline & 144 C & $144 \mathrm{~A}$ & 146 B & $146 \mathrm{~A}$ & 197 B & $148 \mathrm{~A}$ \\
\hline \multirow[t]{2}{*}{$\mathrm{T}_{10}$} & Y-G-G & Y-G-G & Y-G-G & Y-G-G & Y-G-G & $G-G-$ \\
\hline & $144 \mathrm{C}$ & $144 \mathrm{~A}$ & 146 B & 146 B & 148 B & N137 B \\
\hline \multirow[t]{2}{*}{$\mathrm{T}_{11}$} & Y-G-G & Y-G-G & Y-G-G & Y-G-G & Y-G-G & $Y-G-G$ \\
\hline & $144 \mathrm{C}$ & 144 B & $146 \mathrm{C}$ & 146 B & 148 B & $148 \mathrm{~A}$ \\
\hline \multirow[t]{2}{*}{$\mathrm{T}_{12}$} & Y-G-G & Y-G-G & Y-G-G & Y-G-G & Y-G-G & $Y-G-G$ \\
\hline & $144 \mathrm{C}$ & $144 \mathrm{~A}$ & 146 B & $146 \mathrm{~A}$ & 148 B & $148 \mathrm{~A}$ \\
\hline \multirow[t]{2}{*}{$\mathrm{T}_{13}$} & Y-G-G & Y-G-G & Y-G-G & Y-G-G & G-G-G & G-G-N \\
\hline & $144 \mathrm{C}$ & $144 \mathrm{~A}$ & $146 \mathrm{~A}$ & $146 \mathrm{~A}$ & 197 B & $137 \mathrm{~A}$ \\
\hline
\end{tabular}

Indian olive was light green in colour and fruit colour become greenish brown and some times black as the storage period progressed. As a result fruits lost their marketability. First day of storage colour of fruit was in yellowish green group, and gradually changed every day. After 3 days colour of fruits became dark green in yellow green group. Colour change of uncoated fruits was very rapid. After 12 days colour of all the coated fruits was in Y-G-G group except control. In case of uncoated fruits colour of some fruits was in G-G-G 197 B 
group. Again after 15 days worst result was seen in uncoated fruits than the other treatments.

\subsection{Effect of different treatments on changes in TSS (Brix)} during storage of Indian olive

Total soluble solid of the fruits was recorded by hand refractometer. TSS content increased upto certain period of storage and then it was decreased in all the treatments as the storage period progressed (Figure 2). Initial value of TSS was $4.56^{\circ}$ Brix.After 15 days, maximum TSS was observed (5.53 ${ }^{\circ}$ Brix) in guar gum and calcium chloride combined treatment $\left(T_{3}\right)$, whereas it was minimum $\left(4.96^{\circ} \mathrm{Brix}\right)$ in chitosan $\left(T_{10}\right)$ and calcium chloride and chitosan combined fruits $\left(T_{11}\right.$ and $\left.T_{12}\right)$.

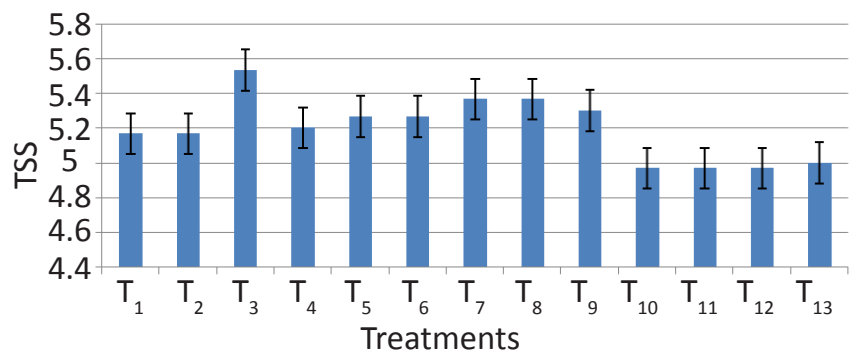

Figure 2: changes in TSS (Brix) during storage of Indian olive

The increase in TSS content during the time of storage might be breakdown of starch (Beaudryet al., 1989) into sugar (Crouch et al., 2003) or hydrolysis of cell polysaccharides (Ben and Gaweda, 1985). Then after completion of hydrolysis of starch no further increase takes place then leads to decline in this parameters as other organic acids are primarily substrate in these parameters (Will et al., 1980).

\subsection{Effect of different treatments on changes in total sugar content during storage of Indian olive}

Observation during storage period revealed that total sugar content (Table 4) increased for a certain period and there after that it was decreased in all the treatment as the storage period progressed. It was increased upto $6^{\text {th }}$ day of storage. At initial day of storage it was $6.35 \%$. At last day of storage $\left(15^{\text {th }}\right.$ day) total sugar per cent was lower than initial percentage in all the treatments. Lowest $(6.04 \%)$ total sugar percentage was seen in fruits which were under control $\left(T_{13}\right)$ whereas highest (6.29\%) total sugar content was seen in guar gum and calcium chloride combined treatment.

The increase in sugar content during the time of storage was due to break down of starch (Beaudry et al., 1989) into sugar (Crouch et al., 2003) and after that started to decrease. As the storage period progressed microbial growth also increased in fruits and for the consumption of sugar content by microbes is the reason of decreasing sugar content.

3.7. Effect of different treatments on changes in reducing sugar content (\%) during storage of Indian olive

Observation during storage period revealed that reducing sugar (Figure 3 ) increased for a certain period after that it was decreased in all the treatments as the storage period
Table 4: Effect of different treatments on changes in total sugar content (Percent) during storage of Indian olive

\begin{tabular}{lccccc}
\hline Treat- & \multicolumn{5}{c}{ Days after storage } \\
\cline { 2 - 6 } ment & 3 & 6 & 9 & 12 & 15 \\
\hline $\mathrm{T}_{1}$ & $7.03^{\mathrm{bc}}$ & $7.38^{\mathrm{a}}$ & $7.21^{\mathrm{ab}}$ & $6.53^{\mathrm{ab}}$ & $6.21^{\mathrm{abc}}$ \\
$\mathrm{T}_{2}$ & $6.79^{\mathrm{cd}}$ & $7.49^{\mathrm{a}}$ & $7.12^{\mathrm{b}}$ & $6.71^{\mathrm{a}}$ & $6.29^{\mathrm{a}}$ \\
$\mathrm{T}_{3}$ & $7.34^{\mathrm{a}}$ & $7.45^{\mathrm{a}}$ & $7.47^{\mathrm{a}}$ & $6.51^{\mathrm{ab}}$ & $6.16^{\mathrm{abcd}}$ \\
$\mathrm{T}_{4}$ & $7.23^{\mathrm{a}}$ & $7.51^{\mathrm{a}}$ & $6.39^{\mathrm{e}}$ & $6.42^{\mathrm{ab}}$ & $6.18^{\mathrm{abcd}}$ \\
$\mathrm{T}_{5}$ & $7.29^{\mathrm{ab}}$ & $7.42^{\mathrm{a}}$ & $6.48^{\mathrm{de}}$ & $6.24^{\mathrm{b}}$ & $6.10^{\mathrm{bcd}}$ \\
$\mathrm{T}_{6}$ & $7.47^{\mathrm{a}}$ & $7.34^{\mathrm{a}}$ & $6.66^{\mathrm{cde}}$ & $6.42^{\mathrm{ab}}$ & $6.20^{\mathrm{abcd}}$ \\
$\mathrm{T}_{7}$ & $6.88^{\mathrm{cd}}$ & $7.28^{\mathrm{abc}}$ & $6.80^{\mathrm{c}}$ & $6.53^{\mathrm{ab}}$ & $6.24^{\mathrm{ab}}$ \\
$\mathrm{T}_{8}$ & $6.74^{\mathrm{d}}$ & $7.16^{\mathrm{abc}}$ & $6.57^{\mathrm{cde}}$ & $6.29^{\mathrm{b}}$ & $6.14^{\mathrm{abcd}}$ \\
$\mathrm{T}_{9}$ & $6.72^{\mathrm{d}}$ & $6.76^{\mathrm{d}}$ & $7.32^{\mathrm{ab}}$ & $6.48^{\mathrm{ab}}$ & $6.19^{\mathrm{abcd}}$ \\
$\mathrm{T}_{10}$ & $6.73^{\mathrm{dcd}}$ & $6.89^{\mathrm{cd}}$ & $6.50^{\mathrm{de}}$ & $6.31^{\mathrm{b}}$ & $6.06^{\mathrm{cd}}$ \\
$\mathrm{T}_{11}$ & $6.79^{\mathrm{cd}}$ & $7.31^{\mathrm{a}}$ & $6.71^{\mathrm{cd}}$ & $6.36^{\mathrm{b}}$ & $6.14^{\mathrm{abcd}}$ \\
$\mathrm{T}_{12}$ & $6.44^{\mathrm{e}}$ & $7.26^{\mathrm{abc}}$ & $6.81^{\mathrm{c}}$ & $6.44^{\mathrm{ab}}$ & $6.17^{\mathrm{abcd}}$ \\
$\mathrm{T}_{13}$ & $6.66^{\mathrm{de}}$ & $6.93^{\mathrm{bcd}}$ & $6.71^{\mathrm{cd}}$ & $6.44^{\mathrm{ab}}$ & $6.04^{\mathrm{d}}$ \\
$\mathrm{SEm} \pm$ & 0.088 & 0.122 & 0.088 & 0.096 & 0.045 \\
$\mathrm{CD}$ & 0.256 & 0.356 & 0.256 & 0.280 & 0.132 \\
$(p=0.05)$ & & & & & \\
\hline & & & & &
\end{tabular}

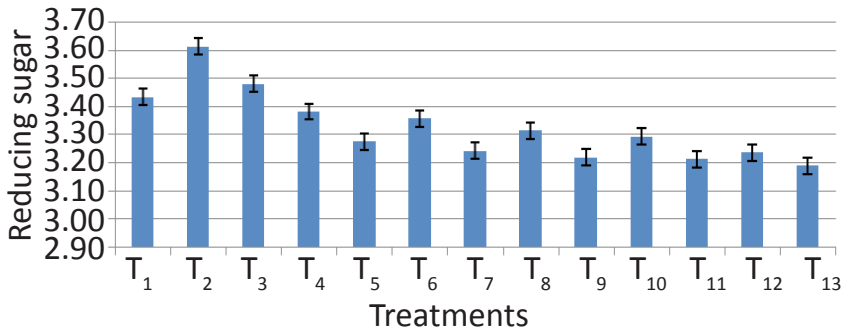

Figure 3: Changes in Reducing sugar content (Percent) during storage of Indian olive

advanced. Initial reducing sugar during storage time was $3.58 \%$. Lowest $(3.19 \%)$ reducing sugar was found in paraffin liquid and calcium chloride combined treatment $\left(\mathrm{T}_{6}\right)$ and chitosan $2 \%$ calcium chloride $2 \%\left(T_{12}\right)$ treatment, where as highest reducing sugar was seen in paraffin liquid $5 \%$ calcium chloride $1 \%\left(\mathrm{~T}_{5}\right)$ treated fruits at the end of storage.

Increasing of reducing sugar is due to break down of starch to sugar. Litchi fruits coated with wax emulsion showed lower amount of reducing sugar in comparision to fruits under control (Garg and Ram, 1972).

3.8. Effect of different treatments on changes in non-reducing sugar content (\%) during storage of Indian olive

Observation during storage period revealed that the nonreducing sugar content (Figure 4) was increased for a certain period and then it was decreased in all the treatments as the storage period advanced. Initial non-reducing sugar content was $2.76 \%$. At the end of storage highest (3.01\%) non-reducing 


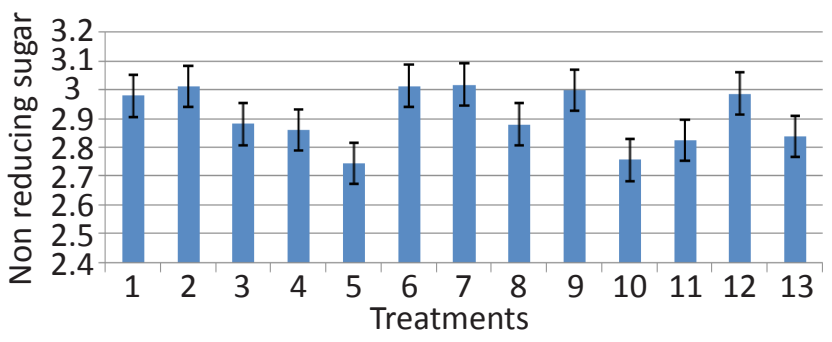

Figure 4: Changes in non reducing sugar content (Percent) during storage of Indian olive

sugar was found in $2 \%$ gum tragacanth treatment $\left(\mathrm{T}_{7}\right)$ followed by paraffin liquid $5 \%+$ calcium chloride $2 \%$ treatment $\left(\mathrm{T}_{6}\right)$ (3.01\%) and lowest $(2.74 \%)$ non-reducing sugar percentage was seen in paraffin liquid and calcium chloride combined treatment $\left(T_{5}\right)$.

3.9. Effect of different treatments on changes in ascorbic acid

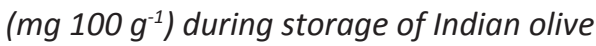

Observation during storage period revealed that ascorbic acid decreased in all the treatments as the storage period advanced. Initial ascorbic acid content was $37.5 \mathrm{mg} 100 \mathrm{~g}^{-1}$ of fruit weight (Table 5). At the end of storage highest (20.86 g $100 \mathrm{~g}^{-1}$ of pulp) ascorbic acid was seen in $\mathrm{T}_{12}$ ( chitosan $2 \%$

Table 5: Effect of different treatments on changes in ascorbic

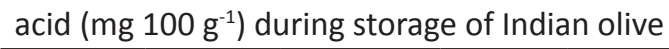

\begin{tabular}{lccccc}
\hline Treat- & \multicolumn{5}{c}{ Days after storage } \\
\cline { 2 - 6 } ment & 3 & 6 & 9 & 12 & 15 \\
\hline $\mathrm{T}_{1}$ & $32.93^{\mathrm{d}}$ & $30.30^{\mathrm{h}}$ & $26.46^{\mathrm{a}}$ & $23.00^{\mathrm{cd}}$ & $19.73^{\mathrm{bc}}$ \\
$\mathrm{T}_{2}$ & $33.30^{\mathrm{bc}}$ & $30.26^{\mathrm{h}}$ & $27.36^{\mathrm{a}}$ & $23.43^{\mathrm{bcd}}$ & $19.36^{\mathrm{c}}$ \\
$\mathrm{T}_{3}$ & $33.36^{\mathrm{bc}}$ & $31.20^{\mathrm{a}}$ & $26.66^{\mathrm{a}}$ & $23.00^{\mathrm{cd}}$ & $19.80^{\mathrm{bc}}$ \\
$\mathrm{T}_{4}$ & $34.16^{\mathrm{ab}}$ & $32.93^{\mathrm{cde}}$ & $26.66^{\mathrm{a}}$ & $23.86^{\mathrm{abc}}$ & $20.30^{\mathrm{abc}}$ \\
$\mathrm{T}_{5}$ & $34.83^{\mathrm{a}}$ & $33.16^{\mathrm{abcd}}$ & $27.30^{\mathrm{a}}$ & $24.03^{\mathrm{ab}}$ & $20.66^{\mathrm{ab}}$ \\
$\mathrm{T}_{6}$ & $34.16^{\mathrm{ab}}$ & $32.53^{\mathrm{e}}$ & $27.10^{\mathrm{a}}$ & $24.23^{\mathrm{ab}}$ & $20.86^{\mathrm{ab}}$ \\
$\mathrm{T}_{7}$ & $35.00^{\mathrm{a}}$ & $33.70^{\mathrm{a}}$ & $27.26^{\mathrm{a}}$ & $24.03^{\mathrm{ab}}$ & $20.13^{\mathrm{abc}}$ \\
$\mathrm{T}_{8}$ & $34.76^{\mathrm{a}}$ & $33.56^{\mathrm{ab}}$ & $26.96^{\mathrm{a}}$ & $24.06^{\mathrm{ab}}$ & $21.16^{\mathrm{a}}$ \\
$\mathrm{T}_{9}$ & $33.73^{\mathrm{bc}}$ & $33.43^{\mathrm{abc}}$ & $26.46^{\mathrm{a}}$ & $24.13^{\mathrm{ab}}$ & $21.10^{\mathrm{a}}$ \\
$\mathrm{T}_{10}$ & $33.43^{\mathrm{bc}}$ & $33.00^{\mathrm{bcde}}$ & $26.93^{\mathrm{a}}$ & $24.03^{\mathrm{ab}}$ & $20.70^{\mathrm{ab}}$ \\
$\mathrm{T}_{11}$ & $33.56^{\mathrm{bc}}$ & $32.63^{\mathrm{de}}$ & $26.96^{\mathrm{a}}$ & $24.60^{\mathrm{a}}$ & $20.66^{\mathrm{ab}}$ \\
$\mathrm{T}_{12}$ & $33.70^{\mathrm{bc}}$ & $31.96^{\mathrm{f}}$ & $27.00^{\mathrm{a}}$ & $24.23^{\mathrm{ab}}$ & $20.86^{\mathrm{ab}}$ \\
$\mathrm{T}_{13}$ & $32.16^{\mathrm{a}}$ & $28.83^{\mathrm{a}}$ & $24.46^{\mathrm{a}}$ & $22.73^{\mathrm{ab}}$ & $18.20^{\mathrm{d}}$ \\
$\mathrm{SEm} \pm$ & 0.272 & 0.190 & 0.297 & 0.276 & 0.373 \\
$\mathrm{CD}$ & 0.793 & 0.554 & 0.865 & 0.802 & 1.085 \\
$(p=0.05)$ & & & & & \\
\hline
\end{tabular}

and calcium chloride $2 \%$ treatment) and $T_{6}$ (paraffin liquid and calcium chloride combined treatment). Lowest ascorbic

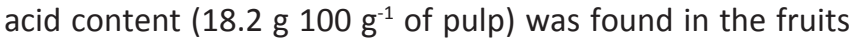
under control $\left(T_{13}\right)$.

From the experiment it is cleared that coated fruits retained more ascorbic acid than the uncoated fruits. Ascorbic acid is a bioactive compound having antioxidant properties (Lata, 2007). Maximum ascorbic acid content was seen at initial day of storage and it was minimum after 15 days of storage. Ascorbic acid content decreased significantly with the increase of storage period. The ascorbic acid in fruits is sensitive to storage temperature or duration and degradation of it enhanced by adverse handling and storage condition like temperature, physical damage (Adisa, 1986). Besides these factors (abiotic), ascorbic acid content can be irreversibly oxidized (Pardio-Sedas et al., 1994), which decreased the edible quality and increased susceptibility to different physiological disorders during storage (Jung and Watkins, 2008).

3.10. Effect of different treatments on changes in tritatable acidity (\%) during storage of Indian olive.

During storage period of Indian olive it was observed that acidity (4.28) increased at a certain period, then it was decreased as the storage period progressed. At initial day of storage it was $2.31 \%$ (Figure 5).

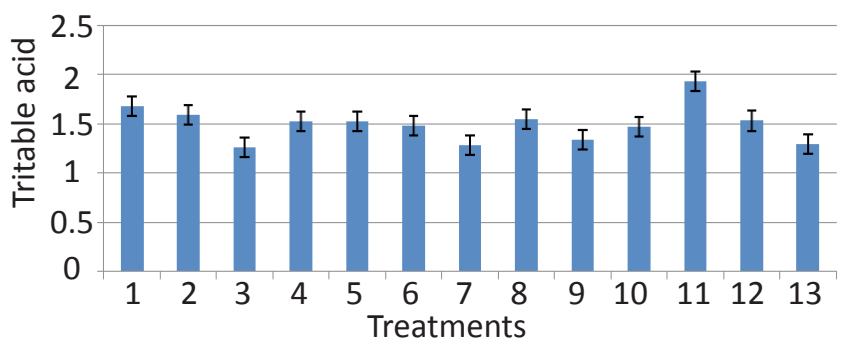

Figure 5: Changes in tritatable acidity (Percent) during storage of Indian olive

At the end of storage lowest (1.25\%) acid content was present in $2 \%$ guar gum and $2 \%$ calcium chloride treatment $\left(\mathrm{T}_{3}\right)$ whereas highest $(1.93 \%)$ was seen in $2 \%$ chitosan and $1 \%$ calcium chloride comined treatment.

The changes of acidity was significantly affected by rate of metabolism (Clarke et al., 2003) mainly respiration which consumed organic acid and for this reason there is decline in acidity during storage ( Ghafir et al., 2009).

\subsection{Effect of different treatments on changes of penetration} force ( $g$ force) during storage of Indian olive.

Fruit firmness is an important criterion for edible quality and market value of Indian olive. It was observed that the amount of penetration force was decrease as the storage period period advanced. Initially it was $2081.2 \mathrm{~g}$ force. At the end of storage maximum (387.633 $\mathrm{g}$ force) penetration force was seen in $2 \%$ guar gum and calcium chloride combined treatment $\left(\mathrm{T}_{2}\right)$ and minimum (241.067 g force) was seen in uncoated fruits.

Fruit firmness is an important criterion for edible quality and market value of apples (De- Ell et al., 2001) and loss of fruit firmness is a serious problem resulting in quality loss (Kovet al., 2005). Fruit firmness is gradually decreased as the storage period progressed due to disassembly of primary cell wall and 
middle lamella structures (Cosgrove et al., 1997) resulted soft and mealy fruit (Gomez et al., 1998).

\section{Conclusion}

Treatments with different wax coating were very useful for prolonging the storage life as well as quality of Indian olive fruits. However, chitosan and combination with other coating agent like calcium chloride significantly improved storage life and fruit quality parameters over other treatments as observed from fruit quality parameters. Thus, storing Indian olive with chitosan coating could be effective to mushrooming marketing and hence farmers income. But, future research is needed to assess the microbial or biological health of fruit.

\section{References}

Adisa, V.A., 1986. The influence of moulds and some storage factors on the ascorbic acid content of orange and pineapple fruits. Food Chemistry 22, 139-146.

Anonymous, 2018. Area and production of Horticulture crops - All India 2016-17 and 2017-18 (First Adv. Est.), National Horticulture Board, Ministry of Agriculture and Farmers Welfare Government of India. page 1, Available from http://nhb.gov.in/statistics/State_Level/201718(1st\%20Adv.\%20Est).pdf.

Baldwin, E.A., Burns, J.K., Kazokas, W., Brecht, J.K., Hagenmaier, R.D., Bender, R.J., Pesis, E., 1999. Effect of two edible coatings with different permeability characteristics on mango (Mangifera indica L.) ripening during storage. Postharvest Biology and Technology 17, 215-226.

Beaudry, R.M., Severson, R.F., Black, C.C., Kays, S.J., 1989. Banana ripening: Implications of changes in glycolytic intermediate concentrations, glycolytic and gluconeogenic carbon flux, and fructose 2, 6-bisphosphate concentration. Journal of Plant Physiology 91, 1436-1444.

Ben, J., Gaweda, M., 1985.Changes of pectic compounds in Jonathan apples under various storage conditions. ActaPhysiologiaePlantarum 7, 45-54.

Bhowmick, N., 2011. Some Lesser Known Minor Fruit Crops of Northern Parts of West Bengal.Acta Horticulturae, 890, 61-63.

Clarke, C.J., McGlone, V.A., Jordan, R.B., 2003. Detection of brownheart in 'Braeburn' apple by transmission NIR spectroscopy. Postharvest Biology and Technology 28, 87-96.

Crouch, I., 2003. 1-Methylcyclopropene (Smart fresh TM) as an alternative to modified atmosphere and controlled atmosphere storage of apples and pears. Acta Horticulture 600, 433-436.

Cosgrove, D.J., Bedinger, P., Durachko, D.M., 1997. Group I allergens of grass pollen as cell wall-loosening agents. Proceedings of the National Academy of Sciences 94, 6559-6564.

De-Ell, J.R., Khanizadeh, S., Saad, F., Ferree, D.C., 2001. Factors affecting apple fruit firmness. Journal of the American Pomological Society 55, 8-27.

De, S., Parikh, K.M., 1985. Medical importance of some common Indian vegetables part 1 . Ancient science of life 4, 232-237.

Dhall, R.K., 2012. Advances in Edible Coatings for Fresh Fruits and Vegetables: A Review. Critical Reviews in Food Science and Nutrition 53, 435-450.

Ghafir, S.A.M., Gadalla, S.O., Murajei, B.N., El-Nady, M.F., 2009. Physiological and anatomical comparison between four different apple cultivars under cold-storage conditions. African Journal of Plant Science 3, 133-138.

Garg, R.C., Ram, H.B., 1972. Effect of different concentrations of wax emulsion on the storage behavior of litchi (Litchi chinensis Sonn). Progressive Horticulture 3, 33-39.

Gomez, C., Fiorenza, F.I., Costell, L., 1998. Perception of mealiness in apples: a comparison of consumers and trained assessors. Zeitschrift fur LebensmittelUntersuchung und-Forschung 207, 304-310.

Jung, S.K., Watkins, C., 2008. Superficial scald control after delayed treatment of apple fruit with diphenylamine (DPA) and 1-methylcyclopropene (1-MCP). Postharvest Biology and Technology 50, 45-52.

Lata, B., 2007. Relationship between apple peel and the whole fruit antioxidant content: year and cultivar variation. Journal of Agricultural and Food Chemistry 55, 663-671.

Mazumder, B.C., Majumder, K., 2003. Determination of chemical constituents. In: Methods on physio - chemical Analysis of Fruits. Daya publishing House, Delhi, 93-139.

Mohamed, S., Khir, S.A., 1993. Maintaining the color, texture and vitamin c of cold-stored pineapples through shrink wrapping and surface-coating with liquid paraffin. Pertanika Journal of Tropical Agricultural Science 16, 201-208.

Pardio-Sedas, V.T., Waliszewski-Kubiak, K.N., Garcia-Alvarado, M.A., 1994. Ascorbic acid loss and sensory changes in intermediate moisture pineapple during storage at 30-40 ${ }^{\circ} \mathrm{C}$. Journal of Food Science and Technology, 551.

Rangana, S., 1997. Manual for analysis of fruit and vegetable products. Tata McGraw Hill Co. Pvt. Ltd., New Delhi, 5.

Sankaran, M., Prakash, Jai., Singh, N.P., Suklabaidya, A., 2006. Wild edible fruits of Tripura. Natural Product Radiance 5, 302-305.

Tharanathan, R., 2003. Biodegradable films and composite coating: past, present and future. Trends in Food Science \& Technology 14, 71-78.

Wijewardane, R.M.N.A., 2013. Application of polysaccharide based composite film wax coating for shelf life extension of guava (var. Bangkok Giant). Journal of PostHarvest Technology 1, 16-21.

Wills, R.B.H., Bembridge, P.A., Scott, K.J., 1980. Use of flesh firmness and other objective tests to determine consumer acceptability of Delicious apples. Australian Journal of Experimental Agriculture 20, 252-256. 